\title{
Méthodologies analytiques pour la spéciation des métaux dans les produits de la mer dans le cadre d'une approche bénéfice/risque (étude CALIPSO)
}

\section{Analytical methodologies for speciation of trace metals in seafood samples in a benefit / risk approach (CALIPSO Study)}

\section{Hervé GARRAUD ${ }^{(1) *}$, Véronique VACCHINA ${ }^{(1)}$, Fabienne SEBY $^{(1)}$, Jean DUMONT ${ }^{(1)}$, Véronique SIROT $^{(2)}$, Thierry GUERIN ${ }^{(2)}$, Jean-Charles LEBLANC ${ }^{(2)}$}

(1) UT2A, Hélioparc Pau Pyrénées, 2, avenue du président Angot, 64053 PAU (2) AFSSA DERNS, 27-31 avenue du général Leclerc, 94701 MAISONS ALFORT

*Auteur à qui adresser la correspondance : Hervé GARRAUD

UT2A, Hélioparc Pau-Pyrénées, 2, avenue du président Angot, 64053 PAU

Tél : 0559806891 - Fax : 0559806367 -E-mail : ut2a@univ-pau.fr

(Reçu le 20 février 2007 ; accepté après modifications le 30 mars 2007)

\section{RÉSUMÉ}

Dans le cadre de l'étude CALIPSO ayant pour objectif de cerner les niveaux d'imprégnation de certains polluants pour de forts consommateurs de produits de la mer, des analyses des teneurs en arsenic, cadmium, mercure et plomb ont été réalisées sur des échantillons de poissons, de crustacés, de mollusques et de produits élaborés ainsi que sur des échantillons de sang humain. L'originalité de l'approche repose en partie sur des analyses de spéciation préalablement développées au laboratoire telles que pour l'arsenic et le mercure. Pour se faire, les méthodologies ont dû être encadrées par des contrôles de qualité internes avant d'être appliquées sur un grand nombre d'échantillons. Ainsi

\section{SUMMARY}

Intheframe ofthe CALIPSO studyconcerning the determination of impregnation levels of some pollutants for seafood consumers, analyses of arsenic, cadmium, mercury and lead were performed on seafood samples and human whole blood. The originality of this approach is partly based on speciation analyses for arsenic and mercury. Analytical methodologies were fully validated and applied to a significative number of samples. Thus, certified reference materials closed to sample matrices were used when possible ( $\mathrm{As}, \mathrm{Cd}, \mathrm{Hg}$ and $\mathrm{Pb}$ total content, and arsenic and mercury organic species). In case of mercury speciation analyses, the isotopic dilution was used in order to correct analytical artefacts such conversion 
lorsque cela a été possible, des échantillons de référence certifiés (CRM) avec une matrice proche de celles étudiées ont été utilisés (détermination des teneurs totales en As, Cd, $\mathrm{Hg}$ et $\mathrm{Pb}$ et teneurs des formes organiques de l'arsenic et du mercure). Dans le cas du mercure, une approche de la dilution isotopique a été mise en ouvre afin d'améliorer les performances analytiques et de pallier certains artefacts analytiques (conversion, correction de rendement d'extraction du méthylmercure). Les paramètres de justesse, de fidélité et les seuils de détection ont été déterminés dans les produits de la mer et le sang humain.

\section{MOTS-CLÉS}

ICP-MS, produits de la mer, sang, spéciation, cadmium, plomb, mercure, arsenic, dilution isotopique.

\section{Introduction}

Dans le cadre de l'étude CALIPSO (étude des Consommations ALimentaires de produits de la mer et Imprégnation aux éléments traces, PolluantS et Oméga 3), une approche bénéfice - risque a été conduite en analysant différents paramètres tels que les teneurs en acides gras essentiels, en polluants organiques persistants et en éléments traces (mercure, plomb, cadmium, arsenic et dérivés organiques de l'étain) dans des produits de la mer consommés par de forts consommateurs (1).

Dans le domaine de l'analyse des éléments traces métalliques, il est aujourd'hui admis que la forme chimique sous laquelle se trouve l'élément permet d'accéder plus finement à sa toxicité éventuelle. Pour certains éléments, tels que l'arsenic et le mercure, la distinction entre les formes chimiques permet d'affiner considérablement la caractérisation du risque en comparant les niveaux entre formes chimiques toxiques (méthylmercure, arsenic inorganique par exemple) et les formes non toxiques comme l'arsénobétaïne. Ainsi, en complément du dosage des teneurs totales en éléments traces, une approche sous l'angle de la spéciation a été conduite afin de mieux connaître les niveaux d'imprégnation de ces éléments lors de la consommation de produits de la mer. Le mercure et l'arsenic étant des éléments d'importance dans les produits de la mer, l'étude CALIPSO s'est focalisée sur des composés comme le méthylmercure, l'arsenic inorganique, et l'arsénobétaïne dans des échantillons de mollusques, de crustacés, de poissons, dans des produits élaborés (paella, surimi) ainsi que dans des échantillons de fluides biologiques (sang, urine).

D'un point de vue analytique, la possibilité de coupler la chromatographie en phase liquide (HPLC) ou plus récemment, la chromatographie en phase gazeuse (GC) à la spectrométrie de masse à plasma induit (ICP-MS) permet la mise en œuvre de méthodes spécifiques et sensibles pour les analyses de spéciation and reaction yield correction. Accuracy, reproducibility and detection limits were the determined in seafood and whole blood samples.

\section{KEY-WORDS}

ICP-MS, seafood, blood, speciation, cadmium, lead, mercury, arsenic, isotopic dilution.

(2-5). Il est alors possible d'accéder à une information plus précise que la simple analyse de teneurs totales. L'acquisition de résultats fiables nécessite cependant la mise en œuvre de méthodologies sous contrôle qualité. Lorsque cela était possible, des matériaux de référence certifiés (CRM) ont été utilisés pour ces validations et ont également permis de déterminer les principaux paramètres statistiques d'une méthode d'analyse (6). Deux types de CRM ont été utilisés lors de cette étude : des tissus de poissons (teneurs totales certifiées en $\mathrm{As}, \mathrm{Cd}, \mathrm{Hg}, \mathrm{Pb}$, teneurs certifiées en méthylmercure, arsénobétaïne et diméthylarsonium), un échantillon de sang (teneurs totales en $\mathrm{As}, \mathrm{Cd}, \mathrm{Hg}, \mathrm{Pb}$ ). Dans le cas des analyses de spéciation du mercure, la méthode de la dilution isotopique a été utilisée (7-9). Ainsi dans le cas d'analyse où il n'existe pas d'échantillons certifiés de référence, comme pour le sang, l'approche par la dilution isotopique permet d'aider à la validation des méthodes d'analyses en introduisant des espèces isotopiquement marquées qui pourront subir lors de la préparation des échantillons et del' analyse, certains artefacts analytiques. Ils permettent au final la correction de certaines erreurs analytiques (10) et améliorent notamment la justesse et la reproductibilité. Il reste néanmoins à évaluer les taux de récupération par des ajouts contrôlés.

Ces modes opératoires ont par la suite été appliqués à 385 échantillons de produits de la mer et 159 échantillons de sang humain et ont permis de collecter un ensemble de données permettant d'émettre des recommandations de consommation grâce à la prise en compte de valeurs de concentrations spécifiques à certaines formes chimiques (1).

\section{Matériels et Méthodes}

\section{Les échantillons}

159 échantillons de produits de la mer et 385 échantillons de sang ont été collectés puis congelés à $-20^{\circ} \mathrm{C}$ avant expédition aux laboratoires d'analyses (1). 
Seuls les produits de la mer ont été par la suite lyophilisés et broyés puis stockés à $+4^{\circ} \mathrm{C}$ dans des sachets en polyéthylène.

Les échantillons de sang sont décongelés avant analyse à température ambiante avant d'être préparés pour le dosage des teneurs totales et les analyses de spéciation.

\section{Dosage des teneurs totales en éléments traces dans les produits de la mer}

Environ $250 \mathrm{mg}$ d'échantillon de produits de la mer sont mis en solution avec $6 \mathrm{~mL}$ d'acide nitrique $(65 \%$ Instra Analysed JT Baker) et $2 \mathrm{~mL}$ de peroxyde d'hydrogène (30\% Instra Analysed JT Baker). Ce mélange est chauffé pendant six heures à $85^{\circ} \mathrm{C}$. Le minéralisat est ensuite ajusté à $50 \mathrm{~mL}$ avec de l'eau ultrapure (Millipore).

L'analyse des teneurs totales en cadmium et plomb est réalisée sur un ICP-MS Elan 6000 (Perkin Elmer Sciex) par étalonnage externe avec reconstitution de la matrice acide. Les échantillons sont dilués dix fois pour accéder à la teneur en arsenic total. Le mercure, en raison des effets mémoire couramment rencontrés en ICP-MS, est analysé par couplage injection dans un flux - ICP-MS (FIA-ICP-MS).

L'échantillon de référence certifié utilisé pour le contrôle de ces analyses est le DORM-2 (Muscle de chien de mer, NRCC Canada).

Les paramètres instrumentaux de cette méthode sont précisés dans le Tableau I.

Tableau I : Paramètres instrumentaux en ICP-MS pour la détermination des teneurs totales en $\mathrm{Cd}, \mathrm{Pb}$, As et $\mathrm{Hg}$.

\begin{tabular}{|c|c|c|}
\hline ICP MS & Perkin Elmer Elan 6000 & $\begin{array}{c}\text { Thermo } \\
\text { Electron X7 }\end{array}$ \\
\hline Puissance & $1300 \mathrm{~W}$ & $1300 \mathrm{~W}$ \\
\hline Type de cônes & Nickel & Nickel \\
\hline Nébuliseur & Flux croisés & Concentrique \\
\hline $\begin{array}{c}\text { Chambre de } \\
\text { nébulisation }\end{array}$ & Scott en Ryton & Bille d'impact \\
\hline $\begin{array}{c}\text { Débit de } \\
\text { nébulisation }\end{array}$ & $0,8-0,9 \mathrm{~L} \cdot \mathrm{min}^{-1}$ & $0,8-0,9 \mathrm{~L} \cdot \mathrm{min}^{-1}$ \\
\hline $\begin{array}{c}\text { Débit gaz } \\
\text { plasmagène }\end{array}$ & $15 \mathrm{~L} \cdot \mathrm{min}^{-1}$ & $15 \mathrm{~L} \cdot \mathrm{min}^{-1}$ \\
\hline $\begin{array}{c}\text { Débit gaz } \\
\text { auxiliaire }\end{array}$ & $0,8 \mathrm{~L} \mathrm{~min}{ }^{-1}$ & $0,8 \mathrm{~L} \mathrm{~min}{ }^{-1}$ \\
\hline $\begin{array}{c}\text { Lentille } \\
\text { d'extraction }\end{array}$ & $\begin{array}{c}\text { Optimisé } \\
\text { quotidiennement }\end{array}$ & $\begin{array}{c}\text { Optimisé } \\
\text { quotidiennement }\end{array}$ \\
\hline $\begin{array}{c}\text { Etalonnage } \\
\text { de la matrice acide à partir } \\
\text { de } 7 \text { étalons } \\
\text { (entre } 0,5 \text { et } 20 \mu \mathrm{g} / \mathrm{L})\end{array}$ & $\begin{array}{c}\text { Ajouts dosés } \\
\text { (entre } 0,05 \\
\text { et } 2 ~ \mu \mathrm{g} / \mathrm{L})\end{array}$ \\
\hline Isotope & $\begin{array}{c}{ }^{75} \mathrm{As},{ }^{113} \mathrm{Cd},{ }^{114} \mathrm{Cd}, \\
{ }^{200} \mathrm{Hg},{ }^{202} \mathrm{Hg},{ }^{206} \mathrm{~Pb},{ }^{208} \mathrm{~Pb}\end{array}$ & $\begin{array}{c}{ }^{75} \mathrm{As},{ }^{113} \mathrm{Cd},{ }^{114} \mathrm{Cd}, \\
\mathrm{Hg},{ }^{202} \mathrm{Hg},{ }^{206} \mathrm{~Pb},\end{array}$ \\
\hline Réplique & 10 & ${ }^{208} \mathrm{~Pb}$ \\
\hline $\begin{array}{c}\text { Temps de } \\
\text { résidence }\end{array}$ & $100 \mathrm{~ms}$ \\
\hline
\end{tabular}

\section{Dosage des teneurs totales en éléments traces dans les échantillons de sang}

$\mathrm{Au}$ préalable, les échantillons sont décongelés puis maintenus dans un bac à ultrasons pendant 20 minutes pour une meilleure homogénéisation.

$250 \mu \mathrm{L}$ sont prélevés et ajoutés à $1 \mathrm{~mL}$ de solution d'acide nitrique 65\% (Instra-Analyzed JT Baker) et $0,25 \mathrm{~mL}$ de peroxyde d'hydrogène (Instra-Analyzed JT Baker). L'échantillon est placé dans un bac à ultrasons pendant 90 minutes puis laissé au repos pendant une nuit. $8,5 \mathrm{~mL}$ d'eau ultrapure (Millipore) sont alors ajoutés avant analyse par ICP-MS Thermo X7 (Thermo Electron).

La quantification est réalisée par la méthode des ajouts dosés à partir d'un échantillon moyen obtenu à partir d'un mélange de l'ensemble des échantillons analysés. Les paramètres instrumentaux pour l'ICP-MS sont proches de ceux donnés dans le Tableau I. Aux niveaux de concentrations qui sont analysés (de $0,05 \mu \mathrm{g} / \mathrm{L}$ à $2 \mu \mathrm{g} / \mathrm{L}$ ), la contamination par effet mémoire du mercure est minime et l'option FIA-ICP-MS n'a pas été retenue. Le bruit de fond est cependant contrôlé lors des séries d'analyses afin de vérifier la contribution du bruit de fond sur les résultats analytiques.

La méthode est contrôlée par l'utilisation d'un échantillon de référence SERONORM I (Sero AS) dont les concentrations en arsenic, cadmium, mercure et plomb sont connus.

\section{Spéciation du mercure dans les produits de la mer}

La prise d'essai est d'environ $250 \mathrm{mg}$ (poids sec). $50 \mu \mathrm{L}$ de solution de méthylmercure marqué avec l'isotope du mercure $202\left(\mathrm{Me}^{202} \mathrm{Hg}\right.$, IRMM) à $0,1 \mu \mathrm{g} / \mathrm{mL}$ sont alors ajoutés. $3,5 \mathrm{~mL}$ d'hydroxyde de tétraméthylammonium (TMAH, Fluka) à $25 \%$ permettent l'extraction des composés du mercure. L'échantillon est irradié sous champ micro-ondes pendant 4 minutes à $20 \mathrm{~W}$ (CEM) dans un flacon en verre de $22 \mathrm{~mL}$.

La dérivation des composés du mercure est réalisée à partir de $2 \mathrm{~mL}$ d'extrait auxquels $5 \mathrm{~mL}$ d'eau ultrapure, $1,2 \mathrm{~mL}$ d'acide acétique glacial (JT Baker), $5 \mathrm{~mL}$ de tampon acétate à $\mathrm{pH}=3,9,1 \mathrm{~mL}$ d'hexane et $1 \mathrm{~mL}$ de solution de tétrapropylborate de sodium à $1 \%$ (Galab) ont été ajoutés. La solution est agitée manuellement pendant 5 minutes puis centrifugée à 3000 tours par minute pendant 5 minutes. La phase organique surnageante est prélevée pour injection en couplage chromatographie en phase gazeuse - spectrométrie de masse à plasma induit (GC-ICP-MS, Thermo X7, Thermo Electron).

Comme pour l'analyse des teneurs totales, l'échantillon de référence certifié utilisé est le DORM-2 (Muscle de chien de mer, NRCC Canada) qui est également certifié 
pour sa teneur en méthylmercure.

L'ensemble des paramètres instrumentaux est regroupé dans le Tableau II. Un chromatogramme présentant la spéciation du mercure dans le DORM-2 est présenté figure 1.

Tableau II : Paramètres instrumentaux pour la spéciation du mercure par couplage $G C-I C P-M S$.

\begin{tabular}{|c|c|}
\hline Colonne GC & $\begin{array}{c}\text { MXT-1 } \\
30 \mathrm{~m} \text { x } 0,53 \mathrm{~mm} \text { diamètre interne } \\
1 \mu \mathrm{m} \mathrm{df}, 100 \% \text { DMPS }\end{array}$ \\
\hline He & $25 \mathrm{~mL} / \mathrm{min}$ \\
\hline Volume d'injection & $1 \mu \mathrm{L}$ splitless \\
\hline $\begin{array}{c}\text { Gradient de } \\
\text { température }\end{array}$ & $\begin{array}{r}60^{\circ} \mathrm{C}-250^{\circ} \mathrm{C} \text { } \mathrm{C} \text { à } 40^{\circ} \mathrm{C} \text { par minute } \\
250^{\circ} \mathrm{C} \text { pendant } 1 \text { minute }\end{array}$ \\
\hline Isotope & ${ }^{199} \mathrm{Hg},{ }^{200} \mathrm{Hg}{ }^{202} \mathrm{Hg}$ \\
\hline
\end{tabular}

Figure 1 : Spéciation du mercure dans le DORM-2 par GC-ICP-MS (suivi des masses 199, 200 et 202).

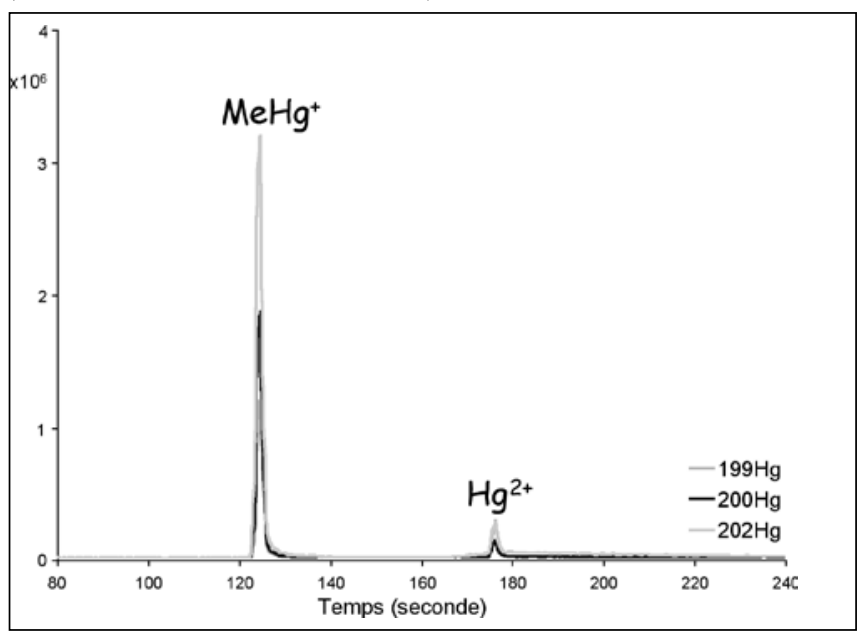

\section{Spéciation du mercure dans les échantillons de sang}

Les échantillons sont décongelés puis soumis à une sonication pendant 10 minutes. $250 \mu \mathrm{L}$ sont prélevés puis mélangés avec $50 \mu \mathrm{L}$ d'une solution de méthylmercure marqué avec l'isotope $202 \mathrm{du}$ mercure $\left(\mathrm{Me}^{202} \mathrm{Hg}\right)$ à $5 \mathrm{ng} / \mathrm{mL}$. L'extraction des composés du mercure est réalisée avec $5 \mathrm{~mL}$ d'acide nitrique $6 \mathrm{~N}$. L'échantillon est placé dans un bac à ultrasons pendant une heure puis centrifugé à 3500 tours par minute pendant 5 minutes.

La dérivation des composés du mercure est réalisée sur $3 \mathrm{~mL}$ d'extrait auxquels $13 \mathrm{~mL}$ d'eau ultrapure, $2 \mathrm{~mL}$ d'ammoniaque, $5 \mathrm{~mL}$ de tampon acétate à $\mathrm{pH}=3,9$, $1,5 \mathrm{mLd}$ 'hexane et $1 \mathrm{~mL}$ de solution de tétrapropylborate de sodium à $1 \%$ ont été ajoutés. La solution est agitée sur une table elliptique pendant 10 minutes (tubes en position horizontale). La phase organique surnageante est prélevée pour injection en couplage chromatographie en phase gazeuse - spectrométrie de masse à plasma induit (GC-ICP-MS). Les paramètres opératoires du couplage GC-ICP-MS sont identiques à ceux décrits dans le Tableau II.

$\mathrm{Au}$ moment de cette étude, aucun échantillon de référence certifié pour la spéciation du mercure dans le sang n'était disponible. La méthode a donc été contrôlée par ajout d'une quantité connue de méthylmercure dans des échantillons réels. Le calcul du taux de récupération de la quantité ajoutée permet de contrôler la stabilité de cette espèce lors des protocoles d'extraction et d'analyse. Par ailleurs, la somme des concentrations en différentes espèces de mercure a été comparée avec la concentration en mercure total déterminée.

\section{Spéciation de l'arsenic dans les produits de la mer}

La prise d'essai est d'environ $250 \mathrm{mg}$ (poids sec). L'extraction des composés organiques et inorganiques de l'arsenic est réalisée avec $5 \mathrm{~mL}$ d'eau ultrapure et $5 \mathrm{~mL}$ de méthanol. Le mélange est agité par ultrasons pendant 1 heure. L'extrait est centrifugé à 2500 tours par minute pendant 5 minutes. Le surnageant est prélevé pour analyse et dilué si nécessaire.

Les composés arséniés sont séparés par HPLC selon un mécanisme d'échange d'anions puis détectés par ICPMS. La quantification est réalisée selon la méthode des ajouts dosés.

La méthode a été validée par l'analyse d'échantillons de référence certifiés (CRM). Deux échantillons certifiés ont été utilisés pour le dosage de l'arsénobétaïne qui est le composé majoritaire (DORM-2, NRCC et BCR 627, IRMM). Le CRM BCR-627 (tissus de thon) a été utilisé pour le contrôle de la teneur en dérivé diméthylarsonium (DMA).

Les paramètres instrumentaux utilisés pour la séparation des composés arséniés sont présentés dans le Tableau III. Un chromatogramme présentant la spéciation de l'arsenic dans le DORM-2 est présenté figure 2.

Tableau III : Paramètres instrumentaux pour la spéciation de l'arsenic par couplage HPLC-ICP-MS .

\begin{tabular}{|c|c|}
\hline Colonne & Dionex AS7 \\
\hline \multirow{2}{*}{ Phase mobile } & $\mathrm{A}: 0,5 \mathrm{mM}$ acétate de sodium \\
& $\mathrm{B}: 25 \mathrm{mM} \mathrm{HNO}$ \\
\hline & $0-3$ min $: 100 \% \mathrm{~A}$ \\
\multirow{3}{*}{ Gradient } & $3-7$ min : gradient jusqu'à $10 \% \mathrm{~B}$ \\
& $10-10 \mathrm{~min}: 18 \% \mathrm{~min}: 6 \% \mathrm{~B}$ \\
& $16-25 \mathrm{~min}: 80 \% \mathrm{~B}$ \\
& $25-35 \mathrm{~min}: 100 \% \mathrm{~A}$ \\
\hline Débit & $1 \mathrm{ml} . \mathrm{min}-1$ \\
\hline \multirow{2}{*}{ Espèces analysées } & Arsénobétaïne, MMA, DMA, As(III), \\
& As(V) \\
\hline
\end{tabular}




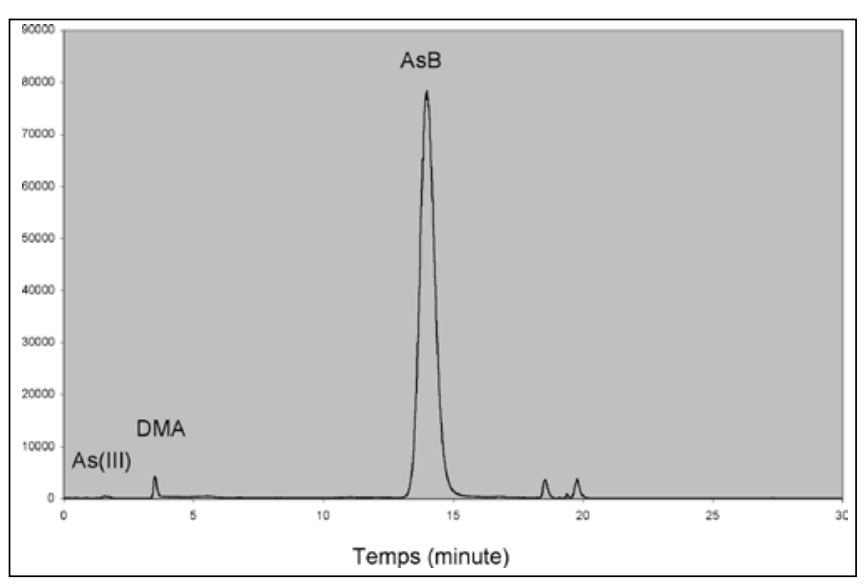

Figure 2 : Spéciation de l'arsenic dans le DORM-2 par HPLC-ICPMS (suivi de la masse 75).

\section{Résultats}

Afin de proposer des protocoles analytiques encadrés par des contrôles qualité, l'utilisation d'échantillons certifiés de référence a permis d'évaluer les principaux paramètres statistiques tels que la justesse, la reproductibilité (contrôle sur plusieurs jours d'analyses) et les seuils de détection. La justesse, exprimée en pourcentage est définie ici comme le rapport entre la concentration mesurée et la concentration certifiée. Cette grandeur permet d'évaluer l'écart entre la valeur mesurée et la valeur certifiée. La reproductibilité, exprimée en pourcentage et évaluée sur plusieurs jours, est définie comme le rapport entre l'incertitude étendue ( 2 fois l'écart type des mesures sur un échantillon de référence certifié) et la moyenne des concentrations mesurées. Cette valeur permet d'évaluer la dispersion des mesures sur plusieurs jours d'analyse. Les paramètres de justesse et de reproductibilité sont suivis lors de chaque série de mesures afin de mettre en évidence une éventuelle dérive des résultats. Les seuils de détection sont évalués à partir de l'ensemble des blancs analysés au cours des séries d'analyses. Ils sont calculés à partir de 3 fois l'écarttype sur le blanc. L'évaluation des seuils de détection a été faite lors de chaque série d'analyse afin de vérifier si les performances de détection restaient dans des ordres de grandeurs acceptables par rapport aux concentrations mesurées dans les échantillons réels.

La méthode est jugée valide lorsque les incertitudes étendues entre la valeur certifiée et la valeur mesurée se recoupent.

Les données concernant les performances analytiques calculées pour les teneurs totales dans les produits de la mer et le sang, pour les concentrations en formes chimiques du mercure et de l'arsenic dans les produits de la mer sont présentées dans le Tableau IV.

\section{Discussion}

\section{Analyses des produits de la mer}

L'analyse des teneurs totales dans les produits de la mer montre des performances acceptables en terme de justesse et de reproductibilité (Tableau IV). Le cadmium et le plomb présentent une variabilité de $18 \%$ et de $25 \%$, respectivement, ce qui est certainement à relier aux faibles niveaux de concentration rencontrés dans l'échantillon certifié DORM-2 et qui sont donc proches des seuils de quantification. Pour l'arsenic, l'analyse en ICP-MS est interférée par la présence de chlore (formation de $\mathrm{ArCl}$ à la masse 75). Le chlore pouvant être présent dans ce type d'échantillons, il a été nécessaire de diluer 10 fois les minéralisats pour atteindre des concentrations en arsenic dans les extraits suffisantes pour une analyse précise mais aussi de réduire la contribution de l'interférence. Cette dernière devient alors négligeable et les concentrations en arsenic restent suffisantes pour une analyse juste et précise comme le montrent les résultats obtenus pour le DORM-2 (Tableau IV). En terme de justesse, la valeur passe de $120 \%$ dans les extraits non dilués à $104 \%$. Pour le mercure, une mesure fiable nécessite le contrôle de l'effet mémoire. Pour cela, l'utilisation de l'injection dans un flux permet l'introduction d'un volume réduit d'échantillon (200 $\mu \mathrm{L})$ minimisant ainsi les temps de rinçage par une solution chloro-nitrique et d'or. Ces conditions, au vu des résultats présentés dans le Tableau IV, apparaissent suffisante pour que la méthode soit validée.

Le dosage des espèces arséniées comme l'arsénobétaïne et le diméthylarsonium (DMA) a été validé par l'analyse du DORM-2. Les justesses sont proches de $100 \%$ pour ces deux espèces. L'analyse du DMA présente une reproductibilité moins bonne que l'arsénobétaïne en raison probablement d'une concentration plus faible. $\mathrm{Ne}$ disposant pas de matériau de référence certifié pour les espèces monométhylarsonium (MMA), As(III) et As(V), les paramètres de validation n'ont pas pu être déterminés pour ces composés. Ces espèces ont néanmoins été analysées afin d'obtenir des données complémentaires sur les échantillons réels. La comparaison des concentrations totales en arsenic avec la somme des concentrations des espèces arséniées quantifiées montre que le bilan reste satisfaisant (figure 3). Cette comparaison amène une confiance supplémentaire dans les analyses menées pour le dosage de l'arsenic total et des espèces arséniées.

L'analyse de spéciation du mercure a été réalisée par dilution isotopique. Cette méthode, considérée comme « primaire » est utilisée comme un moyen de quantification d'une espèce donnée dans un échantillon (7-9). L'addition d'une espèce isotopiquement marquée permet une analyse similaire à celle utilisant un étalon 
Tableau IV : Performances analytiques des protocoles d'analyses.

\begin{tabular}{|c|c|c|c|c|}
\hline \multicolumn{5}{|l|}{ Teneurs totales dans les produits de la mer } \\
\hline Elément & As & Cd & Hg & $\mathbf{P b}$ \\
\hline Valeur certifiée DORM-2 $(\mu \mathrm{g} / \mathrm{g}) *$ & $18,0 \pm 1,1$ & $0,043 \pm 0,008$ & $4,64 \pm 0,26$ & $0,065 \pm 0,007$ \\
\hline Valeur mesurée $(\mu \mathrm{g} / \mathrm{g}) *$ & $18,7 \pm 2,9$ & $0,045 \pm 0,08$ & $4,61 \pm 0,37$ & $0,059 \pm 0,015$ \\
\hline Justesse 16 mesures (\%) sur 4 jours & 104 & 104 & 99 & 90 \\
\hline Reproductibilité 16 mesures (\%) sur 4 jours & 15 & 18 & 8 & 25 \\
\hline Limite de détection ( $\mu \mathrm{g} / \mathrm{g}$ poids sec) & 0,007 & 0,002 & 0,003 & 0,002 \\
\hline \multicolumn{5}{|l|}{ Teneurs totales dans les échantillons de sang } \\
\hline Elément & As & Cd & $\mathbf{H g}$ & $\mathbf{P b}$ \\
\hline Valeur certifiée SERONORM I $(\mu \mathrm{g} / \mathrm{L}) *$ & $1,8 \pm 0,4$ & $0,74 \pm 0,06$ & $2,2 \pm 0,2$ & $27,6 \pm 1,4$ \\
\hline Valeur mesurée $(\mu \mathrm{g} / \mathrm{L})^{*}$ & Non mesuré & $0,63 \pm 0,20$ & $2,3 \pm 0,9$ & $25,2 \pm 5,8$ \\
\hline Justesse 20 mesures (\%) sur 5 jours & & 88 & 106 & 91 \\
\hline Reproductibilité 20 mesures (\%) sur 5 jours & & 32 & 39 & 23 \\
\hline Limite de détection $(\mu \mathrm{g} / \mathrm{L})$ & & 0,03 & 0,3 & 0,2 \\
\hline \multicolumn{5}{|l|}{ Spéciation du mercure dans les produits de la mer } \\
\hline Espèce chimique & \multicolumn{3}{|c|}{ MeHg } & \\
\hline Valeur certifiée DORM-2 $(\mu \mathrm{g} / \mathrm{g}) *$ & \multicolumn{3}{|c|}{$4,159 \pm 0,298$} & \\
\hline Valeur mesurée $(\mu \mathrm{g} / \mathrm{g}) *$ & \multicolumn{3}{|c|}{$4,235 \pm 0,234$} & \\
\hline Justesse 16 mesures (\%) sur 8 jours & \multicolumn{3}{|c|}{102} & \\
\hline Reproductibilité 16 mesures (\%) sur 8 jours & \multicolumn{3}{|c|}{5} & \\
\hline Limite de détection ( $\mu \mathrm{g} / \mathrm{g}$ poids sec) & \multicolumn{3}{|c|}{0,0003} & \\
\hline \multicolumn{5}{|l|}{ Spéciation de l'arsenic dans les produits de la mer } \\
\hline Espèce chimique & \multicolumn{2}{|c|}{ AsB } & MMA & DMA \\
\hline $\begin{array}{ll}\text { Valeur certifiée } & \text { DORM-2 }(\mu \mathrm{g} / \mathrm{g}) * \\
& \text { BCR-627 }(\mu \mathrm{g} / \mathrm{g}) *\end{array}$ & \multicolumn{2}{|c|}{$\begin{array}{l}16,9 \pm 1,1 \\
3,9 \pm 0,2\end{array}$} & Non certifié & $0,15 \pm 0,02$ \\
\hline Valeur mesurée $(\mu \mathrm{g} / \mathrm{g}) *$ & & $\begin{array}{l} \pm 1,2 \\
\pm 0,5\end{array}$ & & $0,16 \pm 0,03$ \\
\hline Justesse 16 mesures (\%) sur 8 jours & & $\begin{array}{l}\text { ORM-2) } \\
\text { R-627) }\end{array}$ & & 107 (BCR-627) \\
\hline Reproductibilité 16 mesures (\%) sur 8 jours & & $\begin{array}{l}\text { RM-2) } \\
\text { R-627) }\end{array}$ & & 19 \\
\hline Limite de détection ( $\mu \mathrm{g} / \mathrm{g}$ poids sec) & & 02 & 0,03 & 0,01 \\
\hline Spéciation du mercure dans le sang & & & & \\
\hline Espèce chimique & & $\mathrm{eHg}$ & & \\
\hline Taux de récupération 12 mesures (\%) sur 4 jours & & 07 & & \\
\hline Reproductibilité 12 mesures (\%) sur 4 jours & & 37 & & \\
\hline Limite de détection $(\mu \mathrm{g} / \mathrm{L})$ & & 95 & & \\
\hline
\end{tabular}

Figure 3 : Comparaison des fréquences d'apparition entre la concentration totale en arsenic et la somme des concentrations des espèces arséniées dans les produits de la mer.

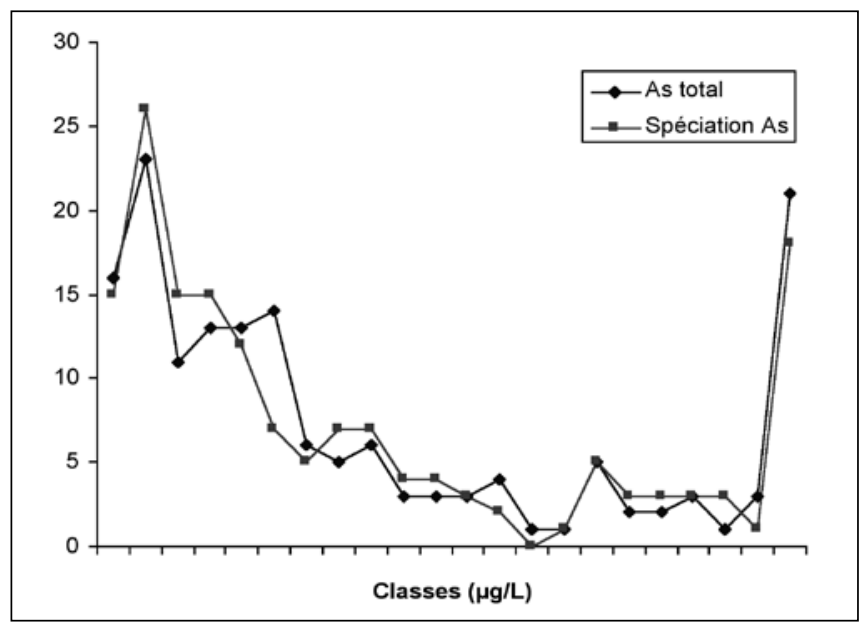

interne. Dans ce cas, la possibilité d'introduire une espèce dont la chimie est identique à celle de la forme recherchée permet de mieux connaître la réactivité et de corriger certains biais analytiques. Avec le couplage GC-ICP-MS, l'analyse de spéciation passe généralement par une phase d'extraction liquide-liquide et de dérivation de l'espèce à analyser. Cette étape permet de transformer les espèces ioniques en espèces volatiles. Seules ces espèces peuvent alors être analysées par chromatographie en phase gazeuse. L'ajout de l'espèce marquée en quantité connue (exemple $\mathrm{Me}^{202} \mathrm{Hg}$ ) permet d'évaluer de manière précise le rendement d'extraction liquide-liquide. Dans le cas du mercure, il sera ainsi possible d'évaluer précisément le rendement d'extraction liquide-liquide lors de la phase de dérivation par l'agent spécifique. Ainsi des facteurs correctifs sont 
appliqués à la concentration calculée permettant ainsi d'atteindre de meilleures justesse et précision dans la mesure. Cependant la dilution isotopique ne permet pas de corriger le rendement d'extraction solide - liquide dans le cas d'échantillons solides (exemples tissus, sols, etc...).

Les résultats obtenus lors de la validation de la méthode sur le DORM-2 montre une justesse de 102\% et une reproductibilité de 5\%. La comparaison des teneurs totales en mercure avec les concentrations en méthylmercure dans les produits de la mer ne présente pas de différence significative et les écarts restent compris dans les erreurs de mesure (figure 4). Cela permet de mettre en évidence que la quasi-totalité du mercure se trouve sous forme de méthylmercure dans les produits de la mer. Pour expliquer ces écarts, plusieurs hypothèses peuvent être retenues :

- L'inhomogénéité des échantillons,

- La matrice (lipide, protéines, éléments interférant la dérivation) peut induire un comportement différent entre le méthylmercure marqué introduit au cours de la préparation des échantillons et le méthylmercure natif présent dans l'échantillon.

Ces hypothèses mettent en évidence que la dilution isotopique, même considérée comme une méthode primaire, ne permet pas de corriger entièrement des artefacts se produisant lors des différentes étapes de la préparation des échantillons comme l'extraction solideliquide ou la réaction de dérivation (propylation).

Figure 4 : Comparaison des fréquences d'apparition entre la concentration totale en mercure et la concentration en dérivés du méthylmercure dans les produits de la mer.

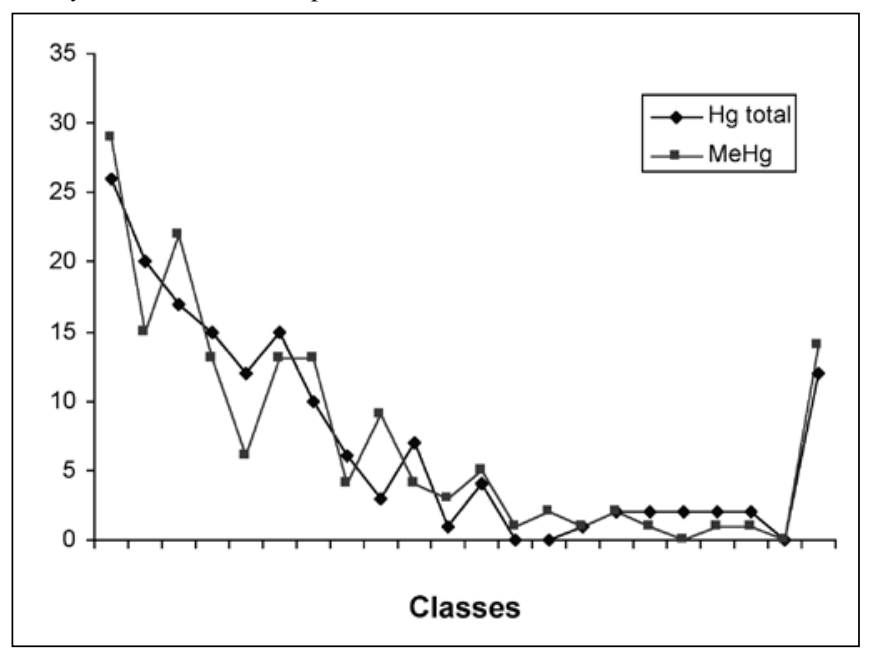

\section{Analyses des échantillons de sang}

L'analyse des échantillons de sang est une analyse problématique en ICP-MS compte tenu de la complexité de la matrice. Les teneurs en sels, en protéines, en lipides, etc... peuvent conduire à une dégradation de l'état de surface des cônes de l'interface entre le plasma et le spectromètre de masse (dépôts de sels, de carbone), mais aussi à une ionisation dégradée des éléments traces analysés par rapport à un milieu aqueux « simple». Afin de prendre en compte ces difficultés, dans un premier temps, nous avons appliqué la méthode développée par Goullé et al (11). Elle consiste en la dilution des échantillons dans un milieu acide nitrique, butanol et triton X100. Au cours d'une série d'analyse, une dérive importante du signal et un niveau de sensibilité insuffisant pour déterminer les concentrations en cadmium, plomb et mercure sont apparus. Nous avons donc choisi une destruction partielle de la matrice par attaque en milieu nitrique, puis une dilution avec de l'eau. Cependant, la présence de l'interférence $\mathrm{ArCl}$ induit une surestimation importante de la concentration en arsenic. Les concentrations mesurées étaient 5 à 10 fois plus importantes que la valeur certifiée. L'ajout d'une quantité connue d'arsenic dans les échantillons certifiés a montré des taux de récupération compris entre 80 et $120 \%$, ce qui montre la limite de l'ICPMS quadripolaire pour des éléments prompts aux interférences polyatomiques. Les valeurs des intensités du signal de l'ajout et de l'échantillon sont additives. L'arsenic ajouté n'est pas affecté par l'interférence et permet alors une évaluation du taux de récupération tout à fait acceptable, or le résultat d'analyse sur l'échantillon est erroné. Dans le cas du sang, une dilution importante, comme dans le cas des produits de la mer, n'est pas possible en raison des faibles concentrations rencontrées (de l'ordre du $\mu \mathrm{g} / \mathrm{L}$ ). Une approche permettant de s'affranchir de cette interférence est alors indispensable. Il peut s'agir de l'utilisation du mécanisme de collision et/ou de réaction présent sur les appareils de dernière génération, ou l'utilisation d'un spectromètre de masse haute résolution ou enfin, d'appliquer la génération d'hydrures afin de séparer la matrice de l'analyte. La première approche semble la plus avantageuse car elle préserve l'approche multi-élémentaire de l'ICP-MS et demeure plus accessible qu'un ICP-MS à secteurs magnétique et électrostatique. L'analyse de l'arsenic dans le sang n'a donc pas pu être validée. Pour les autres éléments, les justesses sont de $88 \%, 106 \%$ et $91 \%$ pour le cadmium, le plomb et le mercure respectivement. La reproductibilité est comprise entre $23 \%$ et $39 \%$. Ces valeurs sont à relier aux faibles concentrations mesurées dans les échantillons dilués (de l'ordre de $0,07 \mu \mathrm{g} / \mathrm{L}$ pour $\mathrm{Cd}$, de $0,2 \mu \mathrm{g} / \mathrm{L}$ et de $2 \mu \mathrm{g} / \mathrm{L}$ pour $\mathrm{Pb}$ ) qui sont proches des limites de quantification de l'appareil ce qui peut induire une variabilité entre différents jours d'analyse. Afin de valider ces résultats, nous avons comparé ces résultats avec ceux d'un autre laboratoire (CHU Angers) qui a analysé les mêmes échantillons 
par ICP-MS. La préparation des échantillons ne nous a pas été communiquée. Afin de comparer les séries de données obtenues par les deux laboratoires, nous avons tracé les fréquences d'apparition des concentrations pour le cadmium (Figure 5), le plomb (Figure 6) et le mercure (Figure 7). La simple comparaison des moyennes et écarts types obtenues ne nous ait pas apparu comme suffisamment pertinente pour juger des biais de mesure entre laboratoires. Nous avons plutôt retenu la distribution des concentrations sur l'ensemble des échantillons afin d'évaluer les méthodes utilisés par les deux laboratoires. La comparaison entre les données obtenues dans cette étude par notre laboratoire et le

Figure 5 : Comparaison des fréquences d'apparition entre laboratoires pour la détermination des teneurs en cadmium dans les échantillons de sang.

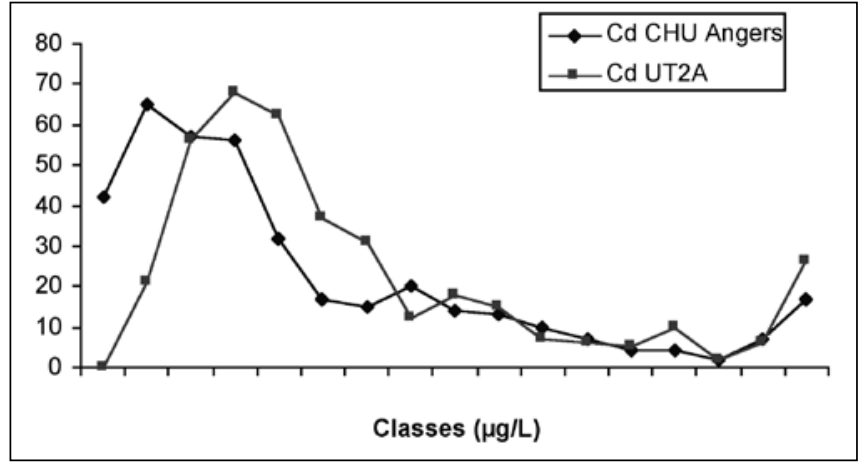

Figure 6 : Comparaison des fréquences d'apparition entre laboratoires pour la détermination des teneurs en plomb dans les échantillons de sang.

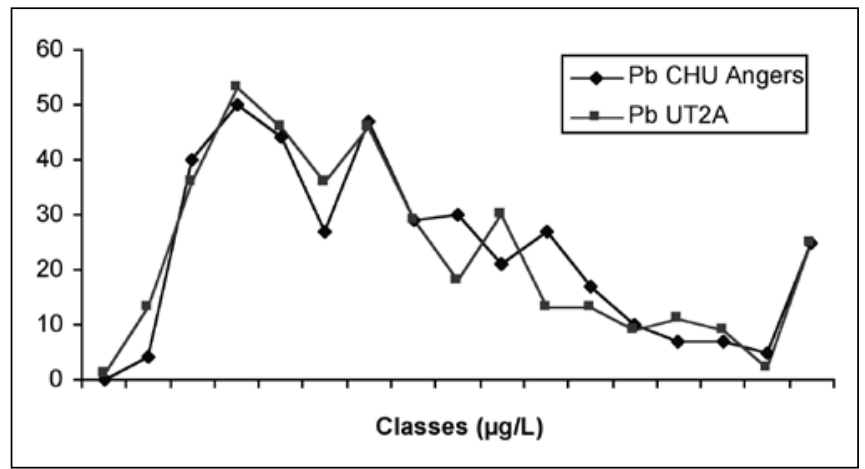

Figure 7 : Comparaison des fréquences d'apparition entre laboratoires pour la détermination des teneurs en mercure et méthylmercure dans les échantillons de sang.

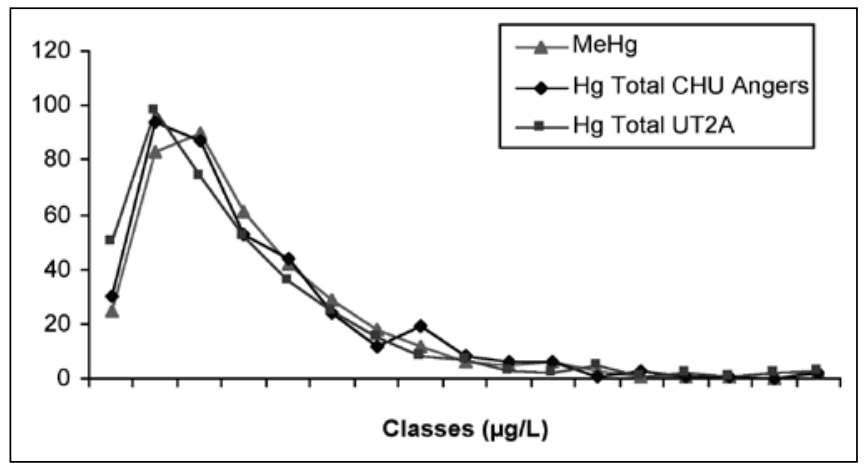

CHU d'Angers montre des résultats concordants. Les moyennes arithmétiques et la dispersion des résultats ne montre pas de différences significatives (Tableau V). Seul le cadmium présente un profil décalé pour les valeurs d'UT2A de $0,2 \mu \mathrm{g} / \mathrm{L}$ entre les deux séries de valeurs (Figure 5). Cet écart demeure dans les erreurs de mesure pour des concentrations considérées comme faibles. Les valeurs moyennes obtenues restent du même ordre de grandeur que celles obtenues par Goullé et al (11) sur 100 sujets témoins.

Tableau $\boldsymbol{V}$ : Comparaison des moyennes et écart types des concentrations mesurées dans les produits de la mer et le sang.

\begin{tabular}{|c|c|c|}
\hline Matrice & Paramètres & Moyenne \pm Ecart type \\
\hline \multirow{2}{*}{$\begin{array}{c}\text { Produit } \\
\text { de la mer }\end{array}$} & Arsenic total & $30 \pm 38 \mu \mathrm{g} / \mathrm{g}$ \\
\cline { 2 - 3 } & $\begin{array}{c}\text { Somme des concentrations des } \\
\text { espèces arséniées }\end{array}$ & $28 \pm 34 \mu \mathrm{g} / \mathrm{g}$ \\
\hline \multirow{2}{*}{$\begin{array}{c}\text { Produit } \\
\text { de la mer }\end{array}$} & Mercure total & $0,53 \pm 0,66 \mu \mathrm{g} / \mathrm{g}$ \\
\cline { 2 - 3 } Sang & Méthylmercure & $0,53 \pm 0,66 \mu \mathrm{g} / \mathrm{g}$ \\
\cline { 2 - 3 } & Cadmium CHU Angers & $0,57 \pm 0,53 \mu \mathrm{g} / \mathrm{L}$ \\
\hline \multirow{2}{*}{ Sang } & Cadmium UT2A & $0,66 \pm 0,58 \mu \mathrm{g} / \mathrm{L}$ \\
\cline { 2 - 3 } & Plomb CHU Angers & $39 \pm 24 \mu \mathrm{g} / \mathrm{L}$ \\
\hline \multirow{3}{*}{ Sang } & Plomb UT2A & $37 \pm 24 \mu \mathrm{g} / \mathrm{L}$ \\
\cline { 2 - 3 } & Mercure total CHU Angers & $3,72 \pm 3,15 \mu \mathrm{g} / \mathrm{L}$ \\
\cline { 2 - 3 } & Mercure total UT2A & $3,49 \pm 3,75 \mu \mathrm{g} / \mathrm{L}$ \\
\cline { 2 - 3 } & Méthylmercure UT2A & $3,74 \pm 3,77 \mu \mathrm{g} / \mathrm{L}$ \\
\hline
\end{tabular}

L'analyse de spéciation du mercure dans les échantillons de sang est très peu citée dans la littérature en raison notamment de la difficulté de traiter la matrice $(3,12)$. De plus, au moment de cette étude, aucun échantillon de référence certifié n'était disponible pour cette matrice. Aujourd'hui, un échantillon de référence certifié en méthylmercure dans du sang de bœuf est proposé par le NIST (SRM 966 level II). L'approche de spéciation du mercure dans le sang reste donc une technique novatrice, pour laquelle la dilution isotopique apparaît être une technique de choix pour augmenter la précision des résultats. Le taux de récupération reste satisfaisant pour ce type d'analyse (Tableau IV). Cependant, il faut noter un coefficient de variation important probablement en raison de la faible sensibilité de cette analyse (Tableau IV). En effet la limite de détection n'est que dix fois inférieure à la concentration moyenne en mercure dans le sang, soit un ratio entre la concentration et la limite de quantification équivalent à un facteur trois. Un gain en sensibilité doit donc être réalisé afin d'améliorer la précision de cette analyse. Pour expliquer cette variabilité entre les concentrations totales et la teneur en dérivé du méthylmercure, on peut reprendre les mêmes hypothèses retenues pour les produits de la mer avec notamment un effet de matrice important pouvant rendre la dérivatisation du mercure relativement inefficace. 


\section{Conclusions}

Les méthodes présentées ici ont permis d'acquérir des données de qualité sur la présence de contaminants dans les produits de la mer sous l'angle de la spéciation grâce, notamment, au développement des couplages entre la chromatographie et l'ICP-MS. L'approche bénéfice - risque peut être alors affinée par l'intermédiaire de la quantification plus précise de ces formes chimiques d'éléments traces d'importance comme l'arsenic et le mercure.

L'application de ces méthodes à des grandes séries d'échantillons présentant des matrices très différentes a nécessité une validation rigoureuse des méthodes d'analyses. Les paramètres tels que la justesse, la reproductibilité et les limites de détection ont été calculés pour l'ensemble des matrices et des paramètres en utilisant des matériaux de référence certifiés ayant une matrice proche des échantillons analysés (poissons, crustacés, mollusques, sang). Ils répondent pour la plupart aux critères d'acceptabilité des méthodes avec des écarts par rapport aux valeurs certifiées inférieures à $15 \%$ ou à une reproductibilité restant dans les ordres de grandeur des valeurs certifiées. Enfin, les seuils de détection obtenus sont suffisamment bas pour obtenir des données fiables pouvant être comparées aux valeurs toxicologiques de référence. L'utilisation d'échantillons de référence certifiés reste néanmoins l'un des prérequis pour ce développement. L'offre reste toutefois insuffisante, notamment pour les formes inorganiques de l'arsenic dans les produits de la mer et pour le méthylmercure dans le sang humain. Ces méthodes s'appuient donc sur des concentrations certifiées d'un nombre restreint de formes chimiques (cas de l'arsenic) ou sur la transposition de méthodes existantes avec l'application de méthodes dites primaires comme la dilution isotopique (spéciation du mercure dans le sang).

La comparaison des concentrations totales avec les approches de spéciation montre que la technique ICPMS permet d'acquérir de nombreuses données à partir d'un grand nombre d'échantillons. Les performances en terme de seuils de détection restent satisfaisantes pour les teneurs couramment rencontrées dans les produits de la mer et le sang humain. Ces outils montrent aussi leur grand potentiel en terme d'analyses de routine.

\section{Remerciements}

Les auteurs tiennent à remercier la Direction générale de l'alimentation du Ministère de l'Agriculture et de la Pêche pour le soutien financier apporté à l'étude CALIPSO, Nadine Flavigny de l'INRA, Patrice Marion et Luc Marchaison pour leur active collaboration au bon déroulement de l'étude, Nadine Frery de l'InVS, Michel
Boisset de l'INSERM et Philippe Verger de l'INRA pour leurs observations et leur aide à l'élaboration du protocole d'étude.

Enfin, les auteurs tiennent tout particulièrement à remercier l'ensemble des personnes enquêtées qui ont accepté de participer à l'enquête CALIPSO, sans lesquelles l'étude n'aurait pu être menée à bon terme.

\section{Références}

1. Leblanc J.C., Sirot V., Volatier J.C., Bemrah Aouachria N., Etude des Consommations alimentaires de produits de la mer et Imprégnation aux éléments traces, polluants et oméga 3, AFSSA, 2006, 160 p.

2. McSheehy S., Szpunar J., Morabito R., Quevauviller P., The speciation of arsenic in biological tissues and the certification of reference materials for quality control. Tr. Anal. Chem. 2003 ; 22 : 191-209.

3. Preudhomme H., Dumont J., Lobinski R., Novelty in analytical approach for the determination of $\mathrm{MeHg}$ and $\mathrm{Hg}^{2+}$ in Human Blood sample by GC-Inductively Coupled Plasma-Isotope Dilution-Mass Spectrometry at the fg level, 2006, en preparation, communication personnelle.

4. Simon S., Tran H., Pannier F., Potin-Gautier M., Simultaneous determination of twelve inorganic and organic arsenic compounds by liquid chromatography - ultraviolet irradiation - hydride generation atomic fluorescence spectrometry, J. Chrom. A. 2004 ; 1024 : 105-113.

5. McSheehy S., Pohl P., Lobinski R. Szpunar J., Investigation of arsenic speciation in oyster test reference material by multidimensionnal HPLC-ICP MS and electrospray tandem mass spectrometry (ES/MS/MS), Analyst. 2001; 126 : 1055-1062.

6. Rivier C., Stumpf C., Labarraque G., Hervouet G., Désenfant M., Priel M., Rouyer M., Seiller M.P., Matériaux de référence et essais d'aptitude : deux outils au service de la qualité des analyses, Spectra Analyse. $2005 ; 256: 33-35$.

7. US EPA Method 6800, Elemental and speciated isotope dilution mass spectrometry, 1998.

8. Rodriguez Martin-Doimeadios R.C., Krupp E, Amouroux D., Donard O.F.X., Application of Isotopically Labeled Methylmercury for Isotope Dilution Analysis of Biological Samples Using Gas Chromatography/ICPMS, Anal. Chem. 2002 ; 74 : 2505-2512.

9. Moreno M.J., Pacheco-Arjona J., Rodriguez-Gonzalez P., Preud'Homme H., Amouroux D., Donard O.F.X., Simultaneous determination of monomethylmercury, monobutyltin, dibutyltin and tributyltin in environmental samples by multi-elemental-species-specific isotope dilution analysis using electron ionisation GC-MS, J. Mass Spect. 2006 ; 41 : 1491-1497.

10. Monperrus M., Krupp E., D. Amouroux, and O. F. X. Donard, Rodriguez Martin-Doimeadios, R.C., Potential and limits of speciated isotope-dilution analysis for metrology and assessing environmental reactivity, Tr. Anal. Chem. $2004 ; 23$ : 261-272. 
11. GoulléJ.P.,MahieuL.,Neveu N.,BouigeD.,Castermant J., Laine G., Nouveau M.P, Gehanne R., Lacroix C., Dosage multi-élémentaire des métaux et métalloïdes dans les milieux biologiques par ICP-MS valeurs usuelles chez 100 témoins, Ann. Toxico. Anal. 2004 ; 16 : 261-268.

12. Bulska E., Emteborg H, Baxter D.C., Frech W., Ellingsen D., Thomassen Y., Speciation of mercury in human whole blood by capillary gas chromatography with a microwave-induced plasma emission detector system following complexometric extraction and butylation, Analyst. $1992 ; 117$ : 657-663. 